\title{
QUI EST DIABÉTIQUE ? LE PATIENT SELON LES MÉDIAS ET LES EXPERTS
}

\author{
Isaac Nahon-Serfaty ${ }^{1}$
}

Qui est le patient? Est-il encore le sujet passif (celui ou celle qui supporte, qui endure la maladie, dans le sens étymologique du mot) ou un sujet actif dans la quête de la guérison, tel que prescrit par les différentes approches de l'empowerment de l'usager ? Ou est-il devenu le 'consommateur éclairé' des produits et des services thérapeutiques ? L'objectif de ce texte est d'explorer les réponses à ces questions depuis le point de vue de deux acteurs qui façonnent les perceptions publiques sur la santé et la maladie, c'est-à-dire les médias et les experts (particulièrement les cliniciens). Un cas en particulier nous servira de terrain pour mieux saisir les enjeux de la représentation du patient, celui de diabète. Topique majeur des discours médicaux et médiatiques, le diabète est devenu un sujet 'hyper communiqué'. Notre analyse repose sur l'analyse des discours médiatiques et experts au Canada à partir d'une démarche qualitative. Le portrait du patient qui émerge dans ces discours sera le résultat d'une lecture des représentations explicites et implicites qui se dégagent de ceux-ci. Ces discours sur le patient diabétique, tels que véhiculés par les médias et les experts, définissent des rôles et des relations entre les acteurs des systèmes de santé, surtout la façon dont les acteurs communiquent et interagissent

1 Professeur adjoint, Département de communication, Université d'Ottawa (Canada). inahonse@uottawa.ca

Recherches en communication, $\mathrm{n}^{\circ} 32$ (2009). 
entre eux et avec les institutions. Cette recherche montre que les discours sur le patient véhiculent à la fois des représentations hautement encadrées, dans lesquelles le patient est surtout un consommateur, et des représentations plus disruptives dans lesquelles le patient est un acteur constamment appelé à agir et réagir dans un environnement de surinformation.

Qui est le patient? Est-il encore le sujet passif (celui ou celle qui supporte, qui endure la maladie, dans le sens étymologique du mot) ou un sujet actif dans la quête de la guérison, tel que prescrit par les différentes approches de l'empowerment de l'usager ? Ou est-il devenu le « consommateur éclairé » des produits et des services thérapeutiques? L'objectif de cette communication - dans la foulée des travaux qui depuis l'anthropologie et la sociologie médicale placent la réflexion sur le patient dans le contexte de l'économie et la culture (Lakoff, 2005 ; Callahan et Wasuna, 2006) et de l'émergence des pandémies globales (McMurray et Smith, 2001) - est d'explorer les réponses à ces questions depuis le point de vue de deux acteurs qui façonnent les perceptions publiques sur la santé et la maladie, c'est-à-dire les médias et les experts, particulièrement les cliniciens et les chercheurs dans le domaine biomédical.

Un cas en particulier nous servira de terrain pour mieux saisir les enjeux de la représentation du patient, celui du diabète. Topique majeur des discours médicaux et médiatiques, le diabète est devenu un sujet 'hyper communiqué'; la maladie est partout, tant par sa condition quasi épidémique que par sa complexité nosologique, c'est-à-dire par l'ensemble de facteurs de risque et de co-morbidités que la définissent (Alexander et al., 2008). C'est donc dans ce contexte d'éclatement et même de fragmentation de discours sur et autour de cette condition, que l'on se demande : qui est diabétique ? La réponse à cette question a des conséquences autant sur la prise en charge des personnes souffrant de diabète, que sur les politiques publiques pour prévenir/traiter le diabète et, surtout, sur les modalités de consommation de produits et services dans le traitement de cette pathologie.

Notre étude repose sur l'analyse des discours médiatiques et experts au Canada à partir d'une démarche qualitative. Le portrait du patient qui émerge dans ces discours sera le résultat d'une analyse des 
représentations explicites et implicites qui se dégagent de ceux-ci. Nous avançons l'hypothèse selon laquelle ces discours sont à la fois des facteurs structurants et le reflet des rapports de pouvoir et économiques dans la sphère sanitaire. Ces discours sur le patient diabétique, tels que véhiculés par les médias et les experts, définissent des rôles et des relations entre les acteurs des systèmes de santé, surtout la façon dont les acteurs communiquent et interagissent entre eux et avec les institutions. Cette recherche montre que les discours sur le patient véhiculent à la fois des représentations hautement encadrées, dans lesquelles le patient est surtout un consommateur, et des représentations plus disruptives dans lesquelles le patient est un acteur constamment appelé à agir et réagir dans un environnement de surinformation.

\section{La construction de l'identité du malade}

Avant d'entrer dans l'analyse des discours sur la personne diabétique, nous allons discuter des notions rattachées à la construction de l'identité sociale du patient. Nous croyons que les discours expert et médiatique participent d'un processus de construction d'identité qui influence les perceptions, les attentes et les rôles du malade dans le cadre institutionnel des systèmes de santé. Toutefois, nous pouvons affirmer avec Carel (2008) que les approches naturalistes et normatives qui cherchent à répondre à la question « Qui est malade ? » ne rendent pas compte de manière satisfaisante de toute la complexité du vécu du patient. Le naturalisme, comme le dit bien Carel (2008, p.8), propose que la maladie est surtout un phénomène biologique qui peut être décrit de façon objective en rendant compte des 'faits' physiques qui la définissent. Pour sa part, l'approche normative, nous rappelle Carel (ibid., p.11), se centre sur la perception sociale du malade, en négligeant la voix individuelle du patient. Elle propose de combler les lacunes de ces approches en abordant la maladie à partir de l'expérience vécue par la personne malade, mais particulièrement comme expérience racontée en suivant les postulats de la phénoménologie (Carel, 2008). Dans ce sens, nous allons partager le même souci de cette philosophe par le langage comme expression d'une identité, mais en privilégiant plutôt l'étude du discours en tant qu'acte d'énonciation sociale, c'est-à-dire en tant que processus de communication.

Comprendre la construction de l'identité du patient comme étant le résultant d'un processus de communication qui s'étale à plusieurs niveaux (communication interpersonnelle, groupale et médiatique), 
implique aussi de s'interroger sur les représentions sociales des maladies. Dans ses essais sur la maladie et ses métaphores, Susan Sontag (1977, 1988) traitait de l'irréductible individualité du vécu humain par rapport à la maladie, une irréductibilité marquée par la conscience d'avoir un corps vulnérable. Or, les ouvrages de Sontag démontrent très clairement comment les métaphores sur les maladies, qui sont des formes explicatives et interprétatives du mal, sont générées dans le social à cause de l'interaction entre la culture, la politique, l'économie et même le domaine militaire. D'une façon plus systématique, l'école de la représentation sociale, initiée par Moscovici (1984, 1989), a étudié comment les conceptions de la santé et de la maladie émergent de la dialectique entre une connaissance qui se veut scientifique et une autre, dite populaire, en produisant une synthèse qui devient donc représentation du collectif (Herzlich, 1969 ; Laplantine, 1989).

L'identité du patient, en tant que construction sociale, est donc le produit d'une tension que Goffman (1963) a bien identifiée en abordant une problématique similaire - celle de la stigmatisation des personnes ayant un handicap - entre une identité sociale virtuelle (virtual social identity), identité assignée par les autres, et une identité sociale réelle (actual social identity), celle associée aux attributs possédés par la personne. Dans le domaine de la maladie, l'identité sociale virtuelle correspondrait bien aux constructions normatives et culturelles du pathologique, tandis que la dite identité sociale réelle répondrait au vécu individuel du malade dans le sens phénoménologique de Carel (2008). Le problème du discours reste surtout celui de la normalisation et des perceptions collectives (le virtuel selon Goffman). Comme l'a bien noté Gilman (1995) dans le cas du Sida, les représentations sociales répondent aussi au besoin de définir les bornes d'un cadre associé à certaines catégories axiologiques (i.e. santé/beauté vs. maladie/laideur) et aux critères de désirabilité sociale.

\section{Méthodologie}

Notre étude combine une analyse des discours médiatique et expert sur le diabète et autres conditions que l'on regroupe dans le cadre du risque cardio-métabolique. Le discours médiatique a été étudié à partir d'un échantillon de nouvelles et articles portant sur le diabète de type 2 publiés dans des journaux canadiens (en anglais et en français) $(\mathrm{n}=280)$. Les nouvelles et articles ont été repêchés dans 
la base de données Canadian Newsstand pour les journaux en anglais The Globe \& Mail (G\&M), Toronto Star (TS) et Vancouver Sun (VS) contenant le mot «diabetes » et qui sont parus entre le 1 janvier et le 31 décembre, $2008(n=210)$. Les articles et nouvelles en français ont été repêchés dans le site web www.cyberpresse.ca $(C P)$ et sont parus au cours de la même période en 2008 à partir d'une recherche du mot clés 'diabète' $(n=70)$. L'étude du discours s'est faite à partir de 11 entrevues en profondeur avec des cliniciens et chercheurs dans trois provinces canadiennes (Colombie Britannique, Ontario et Québec). Les entrevues ont eu lieu en octobre et novembre 2008.

Dans cet article nous adoptons la définition de Lupton (2003, p.142) sur le discours dans le contexte de la communication sanitaire en tant que système de textes, messages, conversations ou dialogues qui sont 'identifiables' (identified) et 'localisables' (located) au sein des structures sociales. De plus, nous souscrivons au point de vue d'Angenot (1988, p.89) selon lequel le discours est l'expression des 'topiques' (en tant que 'lieux communs') ou

[...] l'ensemble des lieux (un topoi) ou présupposés irréductibles du vraisemblable social tels que tous les intervenants des débats s'y réfèrent pour fonder leurs divergences et désaccords parfois violents in praesentia, c'est-à-dire tout le présupposé-collectif des discours argumentateurs et narratifs.

Dans ce sens, les discours médiatique et expert font partie d'un ensemble d'interactions entre institutions, porte-paroles et scientifiques qui finissent par créer ce que Gerstlé (1999, p. 71) a appelé « une réalité de seconde main » dans le domaine médical. Cette perspective intégratrice nous a motivé donc à étudier de façon comparative les deux types de discours, en sachant qu'aujourd'hui on ne peut pas les analyser comme s'ils étaient en vase clos.

Le discours médiatique et expert seront analysés à partir de quatre blocs thématiques autour de la notion de l'identité du patient diabétique : a) l'identité épidémiologique (le profil sociodémographique et géographique du malade) ; b) l'identité étiologique (les causes internes et externes qui affectent le malade) ; c) l'identité pathologique (l'impact sur le malade) ; et d) l'identité thérapeutique (les 'solutions' pour contrôler la maladie, y compris les conséquences nocives des traitements sur le malade). 


\section{Le diabétique selon les médias}

L'identité épidémiologique est surtout définie à partir de la portée de la maladie. Dans ce sens, les médias communiquent une vision ayant un ton presque apocalyptique, car le diabète serait une 'épidémie mondiale'. Le diabétique serait donc un citoyen mondial, 'victime' d'une tendance dans laquelle des facteurs tels l'obésité et le risque cardiovasculaire se combinent pour dessiner un avenir assez sombre :

More than two million Canadians have Type 2 diabetes, a figure that's expected to climb to three million by the end of the decade. A growing number of Canadians also have prediabetes, a condition that occurs when your fasting blood sugar is higher than normal, but not high enough to be diagnosed as diabetes [...]. $(G \& M, 17 / 12 / 08)$

Dans le portrait épidémique dressé par le discours médiatique, le diabétique est souvent une personne pauvre, d'après une représentation qui met l'accent sur les déterminants sociaux de la santé :

Poor women in Ontario are four times as likely to develop diabetes as high-income women. They also are more likely to suffer from high blood pressure, heart disease and depression. (TS, 05/08/08)

L'obésité et même la super obésité sont des facteurs de risque pour développer le diabète. Les médias confirment ainsi un constat médical assez répandu qui fait un lien direct entre le gain de poids et les troubles métaboliques du sucre :

Overweight kids suffer many medical complications, including hypertension, diabetes, high cholesterol, liver disease, pulmonary disorders and social isolation... Obesity experts say there's a direct correlation between heavier children and the amount of TV they watch. Studies show having a TV in a child's bedroom increases their risk of being overweight. (TS, 06/10/08)

Du point de vue de l'identité étiologique, surtout celle qui se dégage du discours médiatique sur les causes du diabète, nous constatons une combinaison entre les facteurs génétiques et les déterminants associés au style de vie. Il y a aurait donc un certain déterminisme 
biologique dans le développement du diabète, déterminisme confirmé par les experts (voir infra) :

If you have a sweet tooth, it won't be found amongst your molars or canines. It's inserted in your DNA instead. The gene may also have implications for a person's risk of getting diabetes. "In humans this gene functions as a glucose sensor in the brain to regulate appetite or food intake," says U of T nutrition expert Ahmed El-Sohemy, the study's principal author. (TS, 14/05/08)

Le portrait étiologique qui suit résume bien une vision polymorphique du diabétique véhiculée par les médias, point de vue confirmé par un certain discours expert (voir infra) :

Previously called non-insulin-dependent diabetes mellitus (NIDDM) or adult-onset diabetes, Type 2 diabetes is the most commonly diagnosed form of the disease [...] Risk factors associated with those age 40 or older, obesity, family history of diabetes, high blood pressure, abnormal cholesterol levels (such as if your HDL or "good" cholesterol levels are 35 or lower or the triglyceride level is 250 or higher), schizophrenia, polycystic ovary syndrome, acanthosis nigricans (darkened patches of skin), previous history of gestational diabetes, history of giving birth to a baby over nine pounds, physical inactivity and ethnicity (those of Aboriginal, Hispanic, Asian or African descent). (TS, 06/11/08)

Par ailleurs, la représentation médiatique reproduit un discours scientifique qui se penche sur certaines déficiences ou excès alimentaires. Par exemple, la déficience en vitamine $\mathrm{D}$ revient souvent comme risque du diabète, un constat souvent accompagné de la recommandation thérapeutique de consommer des suppléments vitaminiques : « Having too little vitamin D is also thought to increase the risk of diabetes, multiple sclerosis, osteoporosis, obesity and certain cancers ». $(G \& M, 31 / 12 / 08)$

Les excès alimentaires sont l'autre visage des mauvaises habitudes alimentaires qui finissent par «causer » le diabète, surtout par leur conséquence la plus visible, soit l'obésité abdominale :

What makes abdominal fat so sinister isn't completely understood. One body of research suggests that visceral fat may make metabolic mischief in its own right, promoting insulin 
resistance, a precursor to diabetes, and inflammation, which may lead to heart disease. $(G \& M, 24 / 04 / 08)$

Le discours médiatique sur la causalité ne situe pas seulement les risques dans la personne en tant qu'individu génétiquement prédisposé, ou ayant certaines caractéristiques (par exemple : obèse, femme enceinte ou pauvre). Le diabète est de plus en plus une condition d'une étiologie diverse associée aussi aux facteurs environnementaux, surtout des facteurs polluants :

Des études ont déjà établi le lien entre de fortes concentrations d'arsenic dans l'eau potable et le diabète de l'adulte, dit de type 2 , mais peu traitent des effets de ce poison à doses plus faibles sur le risque de développer ce type de diabète, ajoutent les chercheurs. $(C P, 19 / 08 / 08)$

Nous observons que les médias se font écho d'une représentation du patient comme un « pré malade », dans le sens d'une identité attachée à l'étape qui précède l'apparition du diabète. Deux notions définissent cette complexification de la représentation du pré-diabétique, c'est-àdire le Syndrome Métabolique (SM) et la résistance à l'insuline :

En découvrant le « chaînon manquant » entre la résistance à l'insuline et les pathologies qui la suivent, le physiologueendocrinologue (le Dr André Marette de l'hôpital Laval) espère qu'il sera possible de développer des médicaments plus efficaces que ceux présentement mis à la disposition des personnes obèses souffrant de diabète de type 2 et de maladies cardiovasculaires [...]. $(C P, 04 / 09 / 08)$

Lorsqu'il s'agit de l'identité pathologique du diabétique, le discours médiatique présente un patient fragilisé face aux autres maladies potentielles, représentation qui confirmerait le caractère polymorphique d'une pathologie 'totalitaire' :

Pour les chercheurs, le diabète pourrait ainsi être la cause de plus de $10 \%$ des cas de tuberculose en Inde et en Chine. Ils soulignent encore qu'accroître les efforts pour diagnostiquer et traiter le diabète pourrait réduire le fardeau global que représente la tuberculose, qui tue environ 1,6 million de personnes chaque année. $(C P, 14 / 07 / 08)$ 
L'identité thérapeutique du patient diabétique véhiculée par les médias dépasse le cadre traditionnel du contrôle du niveau du sucre dans le sang. Il y a là une cohérence entre la complexification de l'étiologie de la maladie et les 'solutions' pour son contrôle. D'une part, la gestion du risque cardiovasculaire du diabétique sert à justifier la prescription 'préventive' des statines pour réduire le cholestérol, prescription qui transforme le désordre métabolique en maladie cardiovasculaire :

Almost all of the more than two million Canadians with diabetes should be taking cholesterol-lowering drugs, according to a major new study. (VS, 11/01/08)

Les médias communiquent aussi un espoir de 'cure' du diabète chez les personnes obèses grâce à la chirurgie bariatrique et autres procédures non chirurgicales, montrant le corps du diabétique comme un terrain d'intervention directe afin de 'renverser' le déficit métabolique grâce aux interventions mécaniques sur le corps du patient :

Les adolescents qui ont recours à la chirurgie pour venir à bout de leur obésité règlent du même coup leur problème de diabète, selon une nouvelle étude menée à petite échelle. $(C P, 29 / 12 / 08)$

Mais le discours thérapeutique des médias est une lame à double tranchant car il est souvent porteur des mauvaises nouvelles sur les effets secondaires des médicaments qui ont des effets paradoxaux sur les patients diabétiques. Le diabétique est, selon ce discours, un malade confronté de plus en plus aux risques thérapeutiques qui représentent une 'crise dans la crise' d'une maladie hors contrôle, comme le montre la nouvelle suivante :

Aggressive diabetes treatment linked to heart attacks, strokes; Experts stunned after higher death rate found among patients. (TS, 08/02/08)

Nous pouvons constater que l'identité du patient qui se dégage du discours médiatique est 'pluriforme' comme 'pluriformes' sont les représentations actuelles sur le diabète (Nahon-Serfaty, 2009). Dans sa virtualité, c'est-à-dire, dans l'identité sociale du diabétique correspond à un modèle de la maladie complexe, multi-causale, multi-thérapeutique et à caractère 'épidémique' ayant une portée mondiale. 


\section{Le diabétique selon les experts}

Le discours expert coïncide avec le discours médiatique en offrant une représentation épidémique du diabète de type 2. Les endocrinologues parlent d'une "épidémie mondiale » (world epidemics), d'une croissance notable de la prévalence chez les adultes et chez les plus jeunes (les enfants et les adolescents), et plus récemment, d'une augmentation des cas de diabète liés à la grossesse. La projection des scénarios prend des allures presque apocalyptiques dans le discours médical. La quantification de l'épidémie met en contexte l'ampleur du problème : «Je ne suis pas convaincu que les Canadiens comprennent qu'au Canada il y a deux millions de diabétiques (de type 2) et que l'on pourrait presque éliminer le diabète au Canada si les gens bougeaient et mangeaient bien », affirme le spécialiste en désordres métaboliques.

Il y a toutefois une fissure dans le consensus. Un clinicien généraliste et chercheur propose un point de vue alternatif : "On pourrait expliquer l'augmentation de la prévalence du diabète comme un résultat direct d'une révision à la baisse de certains critères (lowering thresholds) et plus de dépistage $»^{1}$.

L'identité sociodémographique du patient est souvent étroitement liée à la notion de "famille diabétique ». Cette identité est toutefois problématique pour les familles, particulièrement à cause de l'effet de négation de la part des parents des enfants ou des adolescents qui développent un diabète de type 2 . Ils apprennent avec « surprise » que leurs filles et fils sont malades, "même si $85 \%$ de ces jeunes qui souffrent du diabète ont quelqu'un dans la famille qui en souffre. Ces familles croient qu'il s'agit d'un problème des aînés. Ils me disent, ma mère a eu le diabète quand elle avait 50 ans. Moi, je l'ai eu quand j'avais 40 ou 50 ans. Pourquoi mon enfant en est atteint? », comme le rappelle un endocrinologue.

L'obésité est souvent identifiée dans le discours expert comme la première cause ou le facteur de risque associé à la maladie : « $85 \%$ des patients avec la forme la plus courante du diabète, le diabète de type 2, font de l'obésité, et particulièrement de l'obésité abdominale ", selon un spécialiste dans la physiologie de la condition. Chez les plus jeunes, $95 \%$ de tous les enfants avec un diabète de type 2 étaient obèses

1 Cette réflexion coïncide avec l'étude de Besson et al. (2010) sur la construction de l'épidémie mondiale du diabète et son statut de « menace » pour la santé publique. 
« par définition », observe un endocrinologue, c'est-à-dire qu'ils ont un Indice de Masse Corporelle (IMC) plus élevé que le 95 du percentile dans leur groupe d'âge et leur genre.

La biologie de la maladie confirmerait les rapports de causalité entre obésité et diabète, car dans la plupart des cas, « (les personnes obèses) éprouvent de la difficulté à utiliser le glucose, c'est-à-dire qu'ils ont ce que l'on appelle une intolérance au glucose, un état pré diabétique $»$. Il y a donc dans ce discours une dialectique entre prédisposition génétique et habitudes de vie comme causes du diabète de type 2 . Cette bipolarité des causes et des risques a des conséquences majeures dans la perception de soi chez les patients et la stigmatisation du malade. Une phrase lapidaire prononcée par un chercheur résume bien l'enjeu : « There are people who are just unlucky ».

Les médecins s'interrogent sur la meilleure façon d'aborder le rapport entre les comportements du patient et la maladie sans culpabiliser la personne diabétique. Un cardiologue indique à cet égard : « Il y a beaucoup de facteurs qui déterminent le comportement et nous devons observer le patient sans le blâmer ». Voilà une des clés qui revient dans le discours expert : le problème de la stigmatisation. Tout d'abord, disent certains experts, le fait d'appeler la maladie « diabète » place la condition à l'extérieur du patient, donc le patient ne fait pas le lien entre ses habitudes de vie et l'intolérance au glucose, observe un clinicien. Dans ce sens, le néphrologue précise :

Dans la gestion du diabète, il y a un constat que l'on fait souvent, c'est que les patients ressentent de la honte, car ils croient qu'ils sont responsables de leur maladie, que leur maladie serait le résultat de leurs propres faiblesses personnelles.

La stigmatisation est une lame à double tranchant. Selon un clinicien, lorsqu' on met moins l'accent sur la stigmatisation, c'est-à-dire lorsqu' on veut couper le lien entre la maladie et les habitudes de vie, « on concentre davantage l'attention sur les mesures sanguines (le taux glycémique) et le taux d'hémoglobine glyquée (A1C) que sur le poids ou la quantité d'exercice par semaine ». Cela se traduit donc dans des stratégies thérapeutiques axées sur le contrôle des variables sanguines à travers les médicaments (voir infra).

Le discours médical confirme, comme le fait le discours médiatique par ailleurs, une croissance des complications associées au diabète, particulièrement les co-morbidités cardiovasculaires, les 
maladies des reins et un ensemble de conditions (hypertension, hyperlipidémie, obésité abdominale) que l'on regroupe dans une notion plus large appelée « Syndrome Métabolique » (SM). Dans ce sens, le discours expert se consolide autour d'une redéfinition du diabète et du diabétique. Il ne s'agit plus seulement d'un désordre qui affecte le taux glycémique, mais un ensemble de conditions qui affectent le patient dans sa dimension cardio-métabolique.

Le SM se traduit donc dans une complexification de la gestion de la maladie, complexification bien illustrée par les propos d'un endocrinologue spécialisé dans le diabète chez les plus jeunes qui fait le portrait d'une condition polymorphique :

La chose la plus importante c'est qu'en tant que médecin, quand vous voyez ces enfants vous ne voyez que le diabète, vous voyez la dyslipidémie, vous voyez l'hypertension, vous voyez la dépression, et maintenant nous approchons ces enfants de tous les angles [...] ils ont une obésité, chez les filles c'est les ovaires polycystiques [...] Nous devons voir que ces enfants vont avoir plusieurs risques cardiovasculaires qui vont changer et affecter de façon drastique leur santé.

Or le statut de la personne qui souffre du SM reste ambigu dans la théorie et la pratique médicales. Bien que l'on reconnaisse l'existence du SM (plusieurs répondent rapidement que cela ne change rien car il s'agit d'un concept connu depuis 20 ans), il y a encore de la confusion sur sa définition. La controverse est bien reflétée dans l'analyse d'un chercheur spécialisé dans les désordres métaboliques :

Les médecins savent ce qu'est le SM. Mais si vous leur demandez, savez-vous quoi faire avec ça, là vous allez avoir un grand point d'interrogation. Le SM ce n'est pas tellement compliqué, ce sont les conséquences de l'obésité abdominale, si vous avez de la graisse accumulée dans le mauvais endroit, vous allez faire de la résistance à l'insuline, de l'inflammation, un paquet d'anomalies. Il y a un problème sémantique, commenton appelle cette soupe.

Deux experts affirment que le SM affecte profondément la façon dont les patients se perçoivent eux-mêmes et comment ils conçoivent les risques, les bénéfices et les critères de succès thérapeutique. Un clinicien généraliste indique que « c'est aussi une façon de déterminer 
de façon hâtive quand le patient doit aller voir le médecin, ce qu'ils doivent faire et comment ils vont prendre leurs médicaments ». Un cardiologue précise à cet égard :

D'un point de vue pratique, le traitement du diabète revient aux habitudes de vie, les médicaments oraux, l'insuline, et bien sûr il y a une base théorique, mais je ne suis pas certain que quand je discute avec le patient le jour au jour, je parle de résistance à l'insuline. En faisant cela, je le place encore dans ce modèle de gestion de la maladie et cela crée une distance (entre le patient et la maladie).

Le SM représente une barrière conceptuelle au moment de définir une identité thérapeutique claire pour le médecin et le patient. Un cardiologue spécialiste en patients diabétiques affirme : "L'obésité en soi n'est pas reconnue en cardiologie, on sait que c'est important, ça paraît bien d'en parler, mais dans la vie de tous les jours, les gens (médecins) n'en parlent pas aux patients, ne mesurent pas l'IMC ou le tour de taille».

Le discours expert reconnaît un vide dans les guides thérapeutiques sur le SM. Un clinicien généraliste observe que « pour le diabète, il y a des guides pratiques que les médecins de première ligne et les autres médecins utilisent pour guider leur conduite. Pour ce qui est du SM il n'y a pas encore de guide pratique parce que les gens ne s'entendent pas sur la définition $»$.

Nous constatons encore une fois, comme dans le cas du discours médiatique, que l'identité du patient est polymorphique à cause du caractère 'totalitaire' d'une maladie qui attaque plusieurs organes de la personne diabétique. Les patients qui ont déjà d'autres maladies ou complications associées au diabète n'ont pas toujours conscience des rapports entre la maladie préalable (par exemple, la déficience rénale) et le diabète. C'est le cas des gens hypertendus, et même des gens ayant le diabète qui ont des complications ou des maladies des reins. Dans le cas des gens qui présentent des complications cardiovasculaires associées au diabète, leur identité ne se construit pas autour de la question purement thérapeutique, dans le sens plus traditionnel du terme, mais à partir d'une certaine idée de " qualité de vie ». Un cardiologue précise : «La principale préoccupation des patients c'est la qualité de vie [...] Leur préoccupation principale ce n'est pas de mourir du cœur, mais c'est l'ensemble des complications ». 
La dichotomie entre facteurs environnementaux et conditions structurelles, surtout celles qui découlent de l'organisation des soins de santé, contribue à communiquer une représentation d'un patient pris dans un modèle axé sur l'usage des médicaments et la gestion épisodique des crises « aiguës ». Dans ce cadre, coïncident les avis de plusieurs cliniciens et chercheurs, le patient n'est pas vraiment empowered. Il rentre dans le système et se laisse emporter par une organisation qui va prendre des soins aigus, avec un accent sur les options pharmacologiques dont l'efficacité sur le contrôle glycémique est reconnue. Un cardiologue résume cette situation en disant que « les gens veulent un quick fix, mais les gens ne sont pas prêts à faire un effort pour marcher 10 milles par jour et faire les bons choix alimentaires ».

Le quick fix thérapeutique est aussi un facteur disruptif dans le rapport entre médecin et patient. Les effets secondaires de certains médicaments, particulièrement les nouvelles autour de ces 'crises thérapeutiques', marquent le ton de leur communication. À cet égard, un endocrinologue observe l'effet 'disruptif' de la communication médiatique dans sa pratique médicale :

Les médias sont un monstre (the beast). Ce « monstre » a comme objectif principal d'offrir de la nouveauté. Il y a donc un impératif, c'est de donner ce qui est nouveau. Les nouvelles intéressantes touchent normalement les extrêmes. En d'autres mots, le titre 'Les médicaments contre le diabète marchent toujours' n'attirera pas beaucoup l'attention des lecteurs. Il y a donc deux types d'histoire sur le diabète, normalement sur les médicaments ou sur d'autres approches : d'un côté, 'ce médicament est un miracle', de l'autre coté 'ce médicament vous tue'. Il n'y a rien au milieu.

Le discours expert montre un patient qui se mobilise davantage afin d'exiger des clarifications au médecin sur les médicaments qu'il prend et qui, dans une certaine mesure, devient plus autonome par rapport à sa décision de continuer ou non le traitement. L'exemple sur l'étude qui a trouvé un lien entre la rosiglitazone, un populaire hypoglycémiant oral, et l'augmentation du risque cardiovasculaire, est revenu souvent dans les propos de plusieurs cliniciens et chercheurs. Le néphrologue raconte que « beaucoup de mes patients ont arrêté de prendre le médicament (après avoir entendu la nouvelle) ». Par ailleurs, un cardiologue décrit ce mécanisme de diffusion des études scientifiques qu'il qualifie comme « pervers » : 
On a eu une méta-analyse qui disait que la rosiglitazone était un danger pour les patients et l'on a perdu énormément de temps pour démystifier ça dans le site de Diabète Québec [...] ça a été de la mauvaise science, une méta-analyse mal faite et les gars ont publié ça dans le New England Journal of Medecine (NEJM) - affirme le cardiologue - c'était un peu tendancieux. Non, ce n'est pas vrai que la rosiglitazone augmente le risque cardiovasculaire, parce que dans toutes les études randomisées bien faites ce n'est pas vrai.

Face à la 'crise' médiatique, c'est-à-dire face au caractère disruptif des médias, les médecins expriment une frustration qui découle de leur perte de pouvoir face au patient car ils n'ont plus le monopole de l'information. Un cardiologue signale en commentant le cas du rosiglitazone et les supposés effets cardiovasculaires de ce médicament :

Cela a eu un effet de perte de temps. Cela a eu un effet d'insécuriser les patients et d'une perte de temps incroyable, de répondre aux patients, parce que la nouvelle a été publiée avant que les organismes publics statuent sur le sujet.

Dans le même sens, un endocrinologue décrit la 'nouvelle situation' dans les rapports médecin -patient :

Souvent les patients vont chercher de l'information dans les médias avant qu'ils l'obtiennent du médecin. Et encore pire, le médecin reçoit l'information des médias avant qu'elle arrive par le système médical [...] Il y a donc le risque que le patient soit mieux informé sur ces histoires que le médecin [...] le médecin n'a plus le monopole de l'information qu'il avait, et vous savez, le monopole de l'information c'est $50 \%$ de la médecine.

Par ailleurs, d'autres signalent qu'un patient plus actif dans la quête d'information sur les options thérapeutiques représente une opportunité de communication plus structurée avec le médecin. Un endocrinologue décrit cette situation dans laquelle

Les gens vont arriver avec des coupures de journaux, des articles, et veulent parler des choses qu'ils ont lues. Je trouve cela très bien, de discuter sur ces sujets avec les patients. Et dans d'autres cas, les patients vont voir quelque chose dans les 
médias que je n'ai pas vu quelque chose qui peut rendre l'espoir (sur la maladie).

Pour sa part, un généraliste élabore davantage sa vision 'convergente' (en opposition à la vision 'disruptive') de la communication avec ses patients :

Quand les patients arrivent avec de l'information, je leur dis, montrez-moi ça, qu'est-ce que vous avez lu, pour vraiment valider que les choses qu'ils ont lues, retenues, c'est correct. Moi, je les félicite de s'informer sur leur santé, moi je ne vois pas ça comme une menace. Souvent ils arrivent avec des choses que l'on ne savait pas, on ne peut pas savoir tout dans la vie.

La tension exprimée par les experts entre une communication 'disruptive' (celle causée par la 'crise' du médicament) et une communication 'convergente' (celle qui facilite le dialogue à partir de l'information trouvée dans les médias), est indicative d'une redéfinition des rôles traditionnels dans le cadre du cabinet médical, voire d'une redéfinition des identités.

\section{Conclusion}

L'identité virtuelle du patient diabétique, dans le sens que Goffman donne à cette notion, semble correspondre à une vision purement normative du malade dans la société. Les médias l'identifient comme un sujet avalé par une vague épidémique (discours confirmé par les experts) qui doit faire face à une multiplication des risques, des co-morbidités et un ensemble des cheminements thérapeutiques. Mais l'on observe que dans le discours expert le patient n'est pas seulement le sujet passif qui se laisse « traîner » par les médecins, les industries biomédicales ou les systèmes de santé. Le patient qui émerge de ce discours est un sujet plus actif qui questionne l'efficacité et la sécurité des médicaments. On voit donc se construire une identité qui questionne le pouvoir du médecin, en créant des situations disruptives dans les rapports entre les acteurs dans les organisations de santé. Nous pouvons affirmer que le discours expert rend compte d'une identité sociale réelle du patient (actual social identity selon Goffman) qui contient des éléments souvent ignorés ou minimisés dans l'identité virtuelle communiquée par les médias, qui ont tendance à réduire le patient à une catégorie épidémiologique ou à un sujet soumis aux contraintes des «styles de vie». 
Le discours expert véhicule aussi des nouveaux rapports entre le médecin et le patient qui expriment une tension entre la normalisation dans la définition de la maladie (diabétique, pré-diabétique, syndrome métabolique) et les difficultés pour mesurer ou standardiser les paramètres de cette définition (obésité abdominale, paramètres sanguins, pathologies associées). En tout les cas, les discours, tant médiatiques qu'experts, cherchent la normalisation, pas dans le sens d'obligation ou d'interdiction, mais dans la production d'actes ou de gestes selon un paramètre de normalité.

Or le discours expert est soucieux de ne pas stigmatiser le patient en l'enfermant dans une catégorie qui externalise sa condition, qui la rend étrangère à la personne qui souffre du diabète. Le problème avec cette représentation « non stigmatisante » est qu'elle est en contradiction avec une étiologie du diabète - communiquée par les médias et les experts - qui fait le lien avec l'obésité et même avec une charge génétique familiale. L'identité virtuelle, selon un certain discours, est « stigmatisante » dans la mesure où le diabétique est présenté comme une personne fragile (par exemple, plus à risque du cancer ou de la tuberculose) et soumise à une condition polymorphe qui s'attaque à plusieurs organes.

Le patient « attaqué » par une maladie définie comme « Syndrome Métabolique », c'est-à-dire par la combinaison d'une série de désordres cardio-métaboliques, a une identité thérapeutique multiple qui touche les changements de style de vie (habitudes alimentaires et activité physique), la consommation de plusieurs médicaments et l'option, dans le cas d'obésité sévère, de la chirurgie bariatrique qui pourrait le « guérir » du diabète. Il est aussi sous l'attaque des crises des médicaments, dans une logique identitaire de crise dans la crise, crise de la maladie et crise de la thérapeutique qui devait servir à la contrôler.

L'exploration de l'identité du patient telle que véhiculée par les discours publics, tant par les médias que par les experts, ouvre des nouvelles pistes pour comprendre le rôle de la communication dans la configuration des imaginaires collectifs autour de la santé et la maladie, son impact dans la structuration des institutions sanitaires, et les rapports entre les différents acteurs dans le cadre de systèmes de santé. 


\section{Références}

Alexander, G. C., N. L. Sehgal, R. M. Molone, et R. S. Stafford. (2008). National Trends in Treatment of Type 2 Diabetes Mellitus, 1994 - 2007 Archives of Internal Medecine, 168 (19), 2088-2094.

Angenot, M. (1988). Pour une théorie du discours social. Littérature, 70, 82-90.

Besson R., Bernard, M., Tijou-Traore, A., et I. Gobatto, (2010). Le diabète, une épidémie mondiale? Construction et qualification d'un fait épidémiologique. Dans Chasles, V. (Éd.), Santé et Mondialisation. (pp. 173-191). Lyon : Université Jean Moulin, Lyon 3.

Callahan, D. et, Wasunna, A.A. (2006). Medicine and the Market. Equity v. Choice. Baltimore: Johns Hopkins University Press.

Carel, H. (2008). Illness. The Cry of the Flesh. Stocksfield Hall, Stocksfield: Acumen.

Gerstlé, J. (1999). La santé dans l'agenda. Le médiatique, le public et la politique. Dans Mathien, M. (Éd.), Médias, Santé, Politique. (pp. 63-74). Paris : L'Harmattan.

Gilman, S.L. (1995). Picturing Health and Illness. Images of Identity and Difference. Baltimore and London: The Johns Hopkins University Press.

Goffman, E. (1963). Stigma. Notes on the management of spoiled identity. Englewood Cliffs, N.J.: Prentice-Hall, Inc.

Herzlich, C. (1969). Santé et maladie, analyse d'une représentation sociale. Paris : Mouton \& Co.

Lakoff, A. (2005). Pharmaceutical Reason. Knowledge and Value in Global Psychiatry. Cambridge, UK, Cambridge University Press.

Laplantine, F. (1989). Anthropologie des Systèmes de Représentation de la Maladie. Dans Jodelet, D. (Éd.), Les représentations sociales. (pp. 31-61). Paris : PUF.

Lupton, D. (2003). Medicine as culture. Thousand Oaks, CA : Sage.

Moscovici, S. (1984). The phenomenon of social representations. Dans Farr, R.M. et Moscovici, S. (Eds.), Social representations. (pp. 3-70). London-Paris: Cambridge University Press-Maison des Sciences de l'Homme.

Moscovici, S. (1989) Des représentations collectives aux représentations sociales: élements pour une histoire. Dans Jodelet, D. (Éd.) Les représentations sociales. (pp. 6-86). Paris : PUF.

McMurray, Ch. et Smith, R. (2001). Diseases of Globalization. Socioeconomic Transitions and Health. London/Sterling: Earthscan Publications Ltd.

Sontag, S. (1977). Illnes as Metaphor. New York: Farrar, Strauss and Giroux.

Sontag, S. (1988). AIDS and Its Metaphors. New York: Farrar, Strauss and Giroux. 